\title{
Direct observation of nanoparticle diffusion in cytoplasm of single plant cells realized by photoinjection with femtosecond laser amplifier
}

\author{
Taufiq Indra Rukmana1, Ryohei Yasukuni1, Gabriela Moran2, Rachel Méallet-Renault2, Gilles \\ Clavier3, Tadashi Kunieda4, Misato Ohtani4,5, Taku Demura4 and Yoichiroh Hosokawa1
}

AFFILIATIONS

1 Division of Materials Science, Graduate School of Science and Technology, Nara Institute of Science and Technology, 630-0192 Ikoma, Japan

2 Université Paris-Saclay, CNRS, Institut des Sciences Moléculaires d'Orsay, 91405 Orsay, France

3 Université Paris-Saclay, ENS Paris-Saclay, CNRS, PPSM, 94235 Cachan, France

4 Division of Biological Science, Graduate School of Science and Technology, Nara Institute of Science and Technology, 630-0192 Ikoma, Japan

5 Department of Integrated Biosciences, Graduate School of Frontier Sciences, The University of

Tokyo, 277-8562 Chiba, Japan

a)Author to whom correspondence should be addressed: hosokawa@ms.naist.jp

\begin{abstract}
Diffusion is an important process for molecular transport inside plant cells. Recent advancement in plant physiological study demands verification of the diffusion process at the single cell level. In this work, the real-time intracellular diffusion of nanoparticles in the cytoplasm of single plant cells was realized using photoinjection with femtosecond laser amplifier. The diffusion behavior was analyzed by estimating the diffusion coefficient in cytoplasm. In addition, the effect of particle size to the photoinjection efficiency and diffusion was evaluated. Surprisingly, the intercellular diffusion of nanoparticles between single plant cells was also directly observed.
\end{abstract}

Particle diffusion in cytoplasm is an essential process for plant cells to function properly. The diffusion of functional molecules, such as proteins, nucleic acids, hormones, and nutrients, among others, through cell cytoplasm to the intended position in cells is important to be ascertained in the study of plant physiology.1-4) Recent development shows an increasing interest for the direct observation of the diffusion process at the single cell level, especially to understand molecular transport, biological phase separation, and cell-to-cell communication.5-8) However, there has been a lack of available method to directly observe the diffusion in cytoplasm of single plant cells, particularly for large molecules.

Recently, the photoinjection of large molecules into single intact plant cells using femtosecond ( $\mathrm{fs}$ ) laser amplifier has been reported.9,10) The macromolecules diffused through a pore on the cell wall and membrane into cytoplasm. Considering this breakthrough, the method offers a way to monitor real-time diffusion of macromolecules in cell cytoplasm. In this work, the intracellular diffusion of nanoparticles in single plant cells was directly observed using photoinjection with NIR fs laser amplifier coupled with a confocal laser scanning microscope. The diffusion behavior was assessed by 
estimating the diffusion coefficient in cytoplasm, and the effect of particle size was briefly evaluated. In addition, the direct observation of intercellular diffusion between cells was also demonstrated.

As a representative plant cell sample, we used tobacco BY-2 (TBY-2) cell. Wild-type TBY-2 cells were cultured as described previously.9-12) Two-day old cultured cells were used for the photoporation experiments. 0.5M mannitol was added into the final culture medium to control the osmotic pressure. To partially degrade the cell wall, the cells were incubated in the cellulase and pectolyase enzyme solution in a dark place at $27^{\circ} \mathrm{C}$ for $\left.10 \mathrm{~min} .9-11,13\right)$ After these cell preparations, $100 \mu \mathrm{l}$ of the prepared cell suspensions were placed in a glass-bottom dish $(\mathrm{j}=12 \mathrm{~mm}$, Iwaki). All the experiments were conducted within $6 \mathrm{~h}$ to maintain a healthy cell condition.

Femtosecond laser pulses from a regeneratively amplified fs Ti:Sapphire laser system (SpectraPhysics, Solstice-Ref- MT5W, $800 \mathrm{~nm}, 150 \mathrm{fs}$ ) were led into a laser-scanning confocal microscope (Olympus, IX71FVSF-2) and focused on a TBY-2 cell through a 100x oil-immersion objective lens (Olympus; Plan $N, N A=1.25$ ). A single laser pulse was obtained using a mechanical shutter with a gate time of $1 / 125 \mathrm{~s}$ from pulse trains of $125 \mathrm{~Hz}$. The laser pulse energy through the objective lens was measured with a laser power meter (Ophir, Nova Display-Rohr). A sample was put on a motorized microscope stage (Sigma Koki, E-65GR).

Ultrabright green fluorescent nanoparticles (G-FNPs) made of copolymer of styrene and fluorescent BDPMA (borondipyrromethene (BODIPY) methacrylate) were used for sensitive visualization of nanoparticle injection into TBY-2 cells. The volume of G-FNPs is about 20 times and 400 times larger than average genes (2 MDa) and proteins $(30 \mathrm{kDa})$, respectively.14,15) The G-FNP preparation was described in previous reports.16,17)

The photoinjection and diffusion of G-FNPs were observed by confocal fluorescence imaging. A diode-pumped solid-state (DPSS) laser (Spectra-Physics, PC14763) at $488 \mathrm{~nm}$ wavelength was applied for the excitation. $1 \mu \mathrm{l}$ of the G-FNP solution was added to $100 \mu \mathrm{l}$ of the TBY- 2 cell suspension in the glass-bottom dish, and the sample was put on the microscope stage. All fluorescence images were

captured with the same acquisition parameters.

The representative results of photoinjection of the G-FNPs into single TBY-2 cell and subsequent diffusion of the fluorescent nanoparticles in the cell cytoplasm are shown in Fig. 1. The prepared GFNPs were $80 \pm 2.0 \mathrm{~nm}$ in diameter (d) with concentration of $7.3 \pm 0.34 \times 108$ particles $\mathrm{ml}^{-1}$. A single $20 \mathrm{~nJ}$ fs laser pulse was focused at the contact point of cell wall and membrane. After the single shot irradiation, intracellular diffusion of the G-FNPs was evaluated based on the differential fluorescence intensity $(\Delta \mathrm{I})$ as a function of distance from the laser focal point. The $\Delta \mathrm{I}$ value was obtained by subtracting the mean intensity value before photoporation ( $\mathrm{I}_{\text {before }}$ ) from that of after photoporation (lafter).

$\Delta \mathrm{I}=\mathrm{I}_{\mathrm{after}}-\mathrm{I}_{\text {before. }}(1)$

The temporal evolution of fluorescence intensity is shown in Fig. 2. Fluorescence intensity increased immediately after laser irradiation at the laser focal point $(0 \mu \mathrm{m})$ and reached to half maximum at 45 s. At the distances of $10 \mu \mathrm{m}$ and $20 \mu \mathrm{m}$ from the laser focal point, the fluorescence intensity increased slowly from $45 \mathrm{~s}$ and $60 \mathrm{~s}$ after laser irradiation and reached to half maximum at $120 \mathrm{~s}$ and $150 \mathrm{~s}$, respectively. The $\Delta \mathrm{l}$ value was the highest at the laser focal point. Indeed, the area nearby 
laser focusing position was the brightest part, as shown in Fig. 1. It was suggested that the fluorescent nanoparticles were accumulated at the laser focal point before diffusion in the cell cytoplasm.

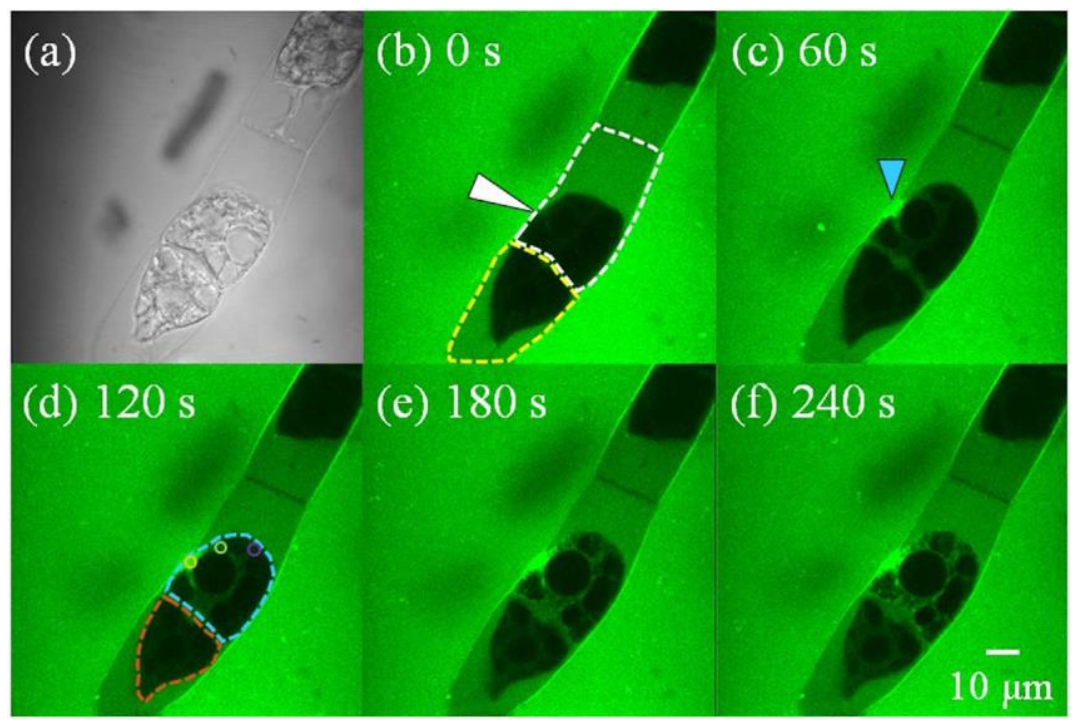

Fig. 1. Confocal fluorescence images of enzyme-and-mannitol treated TBY-2 cells before $(0 \mathrm{~s})$ and after single 20 $n J$ pulse fs laser irradiation $(60,120,180$, and 240 s) under the presence of G-FNPs. The top left image (a) shows the transmission image before laser irradiation. The white and yellow dashed lines in (b) indicate the outlines of a target single cell and its adjacent cell, respectively. The white and blue arrowheads in (b) and (c) indicate the laser focal point and the bright fluorescence observed near the laser focal point after laser irradiation, respectively. The orange, green, and purple circles in (d) indicate the points of fluorescence intensity measurement for the evaluation of intracellular diffusion, approximately at $0 \mu \mathrm{m}, 10 \mu \mathrm{m}$, and $20 \mu \mathrm{m}$ from the laser focal point, respectively. The fluorescence intensity of whole cytoplasm area, surrounded by blue and brown dashed lines in (d) for the target cell and its adjacent cell, respectively, was measured for the evaluation of intercellular diffusion.

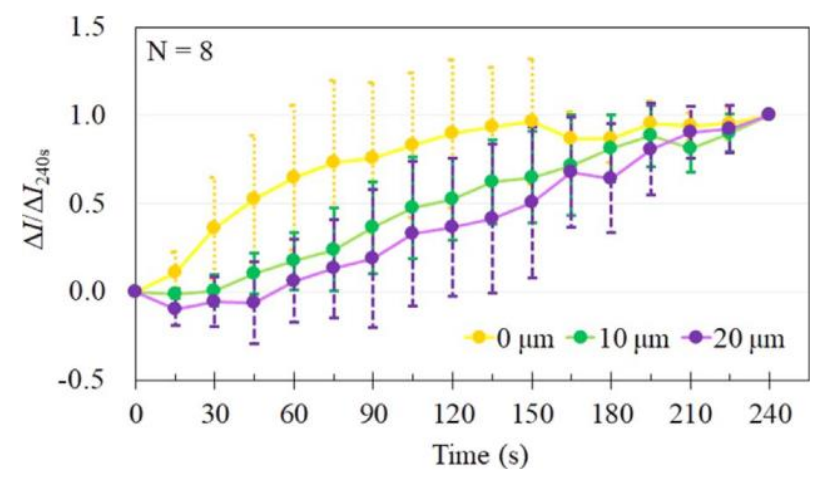

Fig. 2. Temporal evolution of fluorescence intensity in the cytoplasm for enzyme-and-mannitol treated cells after single $20 \mathrm{~nJ}$ pulse fs laser irradiation at various distances from the laser focal point $10 \mu \mathrm{m}, 10 \mu \mathrm{m}$, and 20 $\mu \mathrm{m})$. The graphs are averaged for 8 cells and normalized by the $\Delta l$ at $240 \mathrm{~s}$. The error bar indicates standard deviation.

The diffusion coefficient of the G-FNPs in the cytoplasm was estimated with this equation.18,19)

$$
M S D=2 n D t .(2)
$$

MSD is the mean squared displacement $\left(|\Delta x|^{2}\right)$, with $\Delta x$ as the distance between each measurement point; $\mathrm{n}$ is the dimension of particle movement (3); $\mathrm{D}$ is the diffusion coefficient; and $\mathrm{t}$ is the time 
differential between each point to reach the half maximum intensity. The calculated diffusion coefficients of the G-FNPs in cytoplasm were about $0.2 \mu \mathrm{m} 2 \mathrm{~s}-1$ and $0.6 \mu \mathrm{m} 2 \mathrm{~s}$ for 0-10 $\mu \mathrm{m}$ (D0-10) and 10-20 $\mu \mathrm{m}$ (D10-20), respectively. The D0-10 value was lower than the D10-20 value, confirming that the initial nanoparticle diffusion in cytoplasm, from 0 to $10 \mu \mathrm{m}$, was slower than the later diffusion, from 10 to $20 \mu \mathrm{m}$. Beside nanoparticle accumulation at the laser focal point, the different value of the diffusion coefficient implied that there might be a biological phase separation in the cytoplasm. Meanwhile, the nanoparticle diffusion coefficient in water (Dw), calculated with StokesEinstein equation,20,21) was about $6.1 \mu \mathrm{m} 2 \mathrm{~s}_{-1}$, suggesting that the nanoparticle diffusion in cytoplasm was much slower than in water.

The effect of particle size to the photoinjection efficiency was also examined, as summarized in Fig. 3. Beside the GFNPs $(d=80 \mathrm{~nm})$, other objects with different particle sizes were used: fluorescein isothiocyanate (FITC) conjugated with $20 \mathrm{kDa}$ dextran (FITC-20k), d = $3.2 \mathrm{~nm}$; FITC conjugated with 2 MDa dextran (FITC-2M), $d=26.7 \mathrm{~nm}$; and red fluorescent nanoparticles (R-FNPs), $d=110 \mathrm{~nm}$. The diameter of dextran molecules was calculated with this formula.22,23)

$$
S R[n m]=0.81 x(M W[k D a])^{0.46} .(3)
$$

$\mathrm{SR}$ is the Stokes radius in $\mathrm{nm}$ and MW is the molecular weight in $\mathrm{KDa}$. Smaller particles were injected more efficiently into cell cytoplasm.24) The diffusion through a similar sized pore became slower with increasing particle size.25) The maximum size of injected particles into enzyme-and-mannitol treated cells after irradiation with single $20 \mathrm{~nJ}$ pulse from fs laser amplifier was estimated between $80 \mathrm{~nm}$ and $110 \mathrm{~nm}$ in diameter.

Interestingly, in Fig. 1, we found that the nanoparticles also diffused into the adjacent cell attached to the target cell. To ascertain this, the differential fluorescence intensities before and after laser irradiation in the target cell and adjacent cell were also measured as shown on Fig. 4 . In this case of intercellular diffusion, the whole cytoplasm area of the target and adjacent cells was chosen as a region of interest. It turned out that the fluorescence intensity also increased in the adjacent cell, even though it was lower than that of the target cell. There are many tunnels that connect the cytoplasm between plant cells, called plasmodesmata. Plasmodesmata typically have a diameter of about 50 to $60 \mathrm{~nm}$ in tobacco cells.26) The plasmodesmata could be closed or enlarged in response to cell conditions, such as turgor pressure. In this case, turgor pressure was decreased and the plasmodesmata might be enlarged.27) In addition, the enzyme treatment might have some effects in the enlargement of plasmodesmata, although it still needs to be verified.28) Nevertheless, the gradual diffusion of $80 \mathrm{~nm}$ nanoparticles through this tunnel into the adjacent cell was observed. 

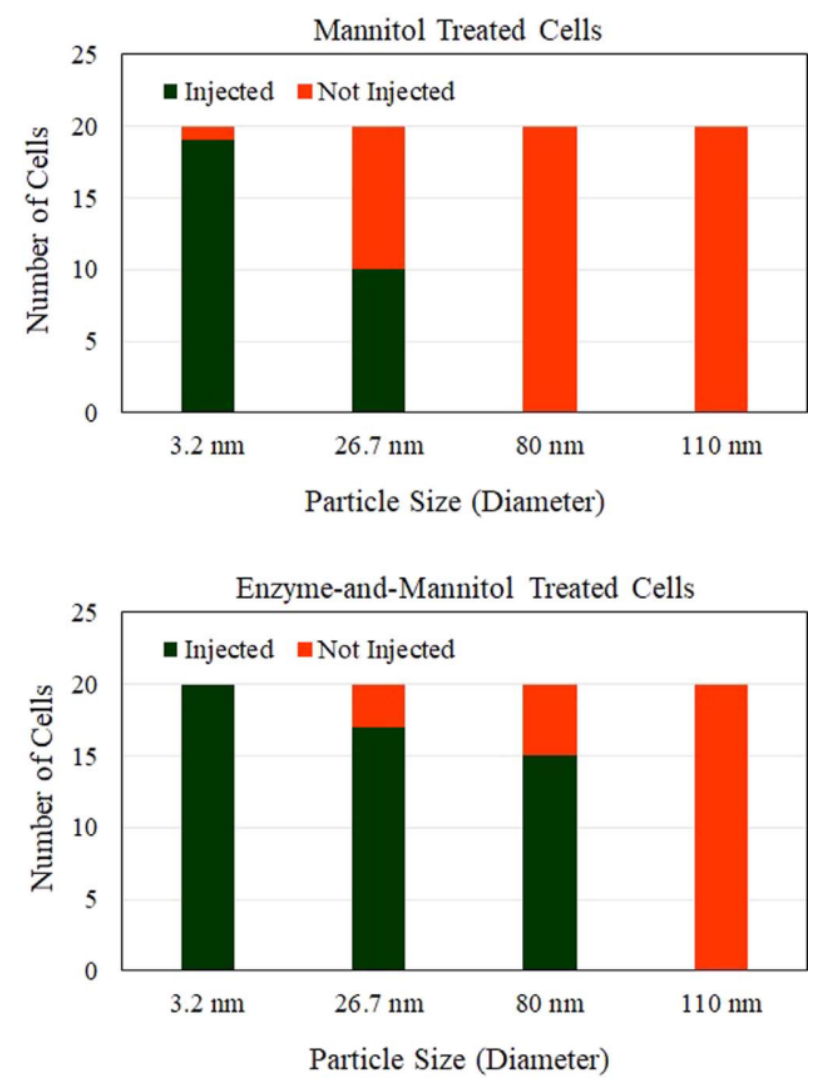

Fig. 3. Photoinjection efficiencies into (a) mannitol treated and (b) enzyme-and-mannitol treated tobacco BY-2 cells after irradiation with single $20 \mathrm{~nJ}$ pulse from fs laser amplifier under presence of objects with different particle sizes: FITC-20k $(d=3.2 \mathrm{~nm})$, FITC-2M $(d=26.7 \mathrm{~nm})$, GFNPs $(d=80 \mathrm{~nm})$ and $R-F N P s(d=110 \mathrm{~nm})$.

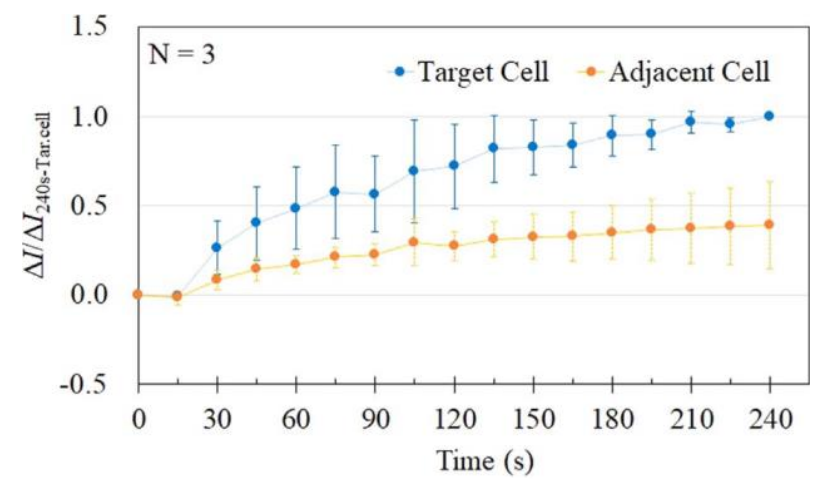

Fig. 4. Temporal evolution of fluorescence intensity in the cytoplasm for enzyme-and-mannitol treated target cells and their adjacent cells after single $20 \mathrm{~nJ}$ pulse fs laser irradiation. The graphs are averaged for three cells and normalized by the $\Delta l$ at $240 \mathrm{~s}$ of the target cell value. The error bar indicates standard deviation.

In previous studies, the movement of nanoparticles in plant organs is analyzed indirectly by measuring the concentration of nanoparticles in leaves and stems after submerging roots or spraying leaves with the nanoparticle suspension.29-31) In contrast, the intercellular diffusion of nanoparticles between single plant cells was directly observed in this work, offering a real-time analysis, which is very promising for the study of plant physiology, particularly to examine molecular transport and cell-to-cell communication. 
In conclusion, the direct observation of intracellular and intercellular diffusion of nanoparticles in the cytoplasm of single plant cells was realized by photoinjection utilizing fs laser amplifier. The ability to observe and evaluate such processes is a major development in the study of plant physiology, especially at the single cell level.

Acknowledgments This work was supported in part by the Grant-in-Aid for Scientific Research (C) (No. JP18042069), Scientific Research on Innovative Areas (No. JP18H05493) from the Japan Society for the Promotion of Science (JSPS). The collaboration between Japan and France was supported by JSPS and MEAEMESRI under the Japan - France Integrated Action Program (PHC Sakura 40951TG).

\section{REFERENCES}

1) U. Neumann, F. Brandizzi, and C. Hawes, Ann. Bot. 92, 167 (2003).

2) T. Tzfira, Y. Rhee, M.-H. Chen, T. Kunik, and V. Citovsky, Annu. Rev. Microbiol. 54, 187 (2000).

3) S. Depuydt and C. S. Hardtke, Curr. Biol. 21, R365 (2011).

4) I. Aibara and K. Miwa, Plant Cell Physiol 55, 2027 (2014).

5) M. Libault, L. Pingault, P. Zogli, and J. Schiefelbein, Trends Plant Sci. 22,949 (2017).

6) V. Demidchik, S. Shabala, S. Isayenkov, T. A. Cuin, and I. Pottosin, NewPhytol. 220, 49 (2018).

7) C. L. Cuevas-Velazquez and J. R. Dinneny, Curr. Opin. Plant Biol. 45, 68 (2018).

8) M. Kitagawa and D. Jackson, Plants 6, 12 (2017).

9) T. I. Rukmana, G. Moran, R. Méallet-Renault, M. Ohtani, T. Demura, R. Yasukuni, and Y. Hosokawa, Sci. Rep. 9, 17530 (2019).

10) T. I. Rukmana, G. Moran, R. Méallet-Renault, G. Clavier, T. Kunieda, M. Ohtani, T. Demura, R. Yasukuni, and Y. Hosokawa, APL Photonics 5, 066104 (2020).

11) H. Schinkel, P. Jacobs, S. Schillberg, and M. Wehner, Biotechnol. Bioeng. 99, 244 (2008).

12) T. Nagata, Y. Nemoto, and S. Hasezawa, Int. Rev. Cytol. 132, 1 (1992).

13) Y. Tamaru, S. Ui, K. Murashima, A. Kosugi, H. Chan, R. H. Doi, and B. Liu, Appl. Environ. Microbiol. 68, 2614 (2002).

14) E. Dauty, J. P. Behr, and J. S. Remy, Gene Ther. 9, 743 (2002).

15) N. S. Bhise, R. B. Shmueli, J. Gonzalez, and J. J. Green, Small 8, 367 (2012).

16) C. Grazon, J. Rieger, R. Méallet-Renault, G. Clavier, and B. Charleux, Macromol. Rapid Commun. 32, 699 (2011).

17) C. Grazon, J. Rieger, R. Méallet-Renault, B. Charleux, and G. Clavier, Macromolecules 46, 5167 (2013).

18) A. M. Berezhkovskii, Y. U. A. Makhnovskii, and R. A. Suris, Phys. Lett. A 150, 296 (1990).

19) B. S. Schuster, J. S. Suk, G. F. Woodworth, and J. Hanes, Biomaterials 34, 3439 (2013).

20) A. Einstein, Ann. Phys. 17, 549 (1905).

21) E. A. Mun, C. Hannell, S. E. Rogers, P. Hole, A. C. Williams, and V. V. Khutoryanskiy, Langmuir 30, 308 (2014).

22) K. A. Granath, J. Colloid Sci. 13, 308 (1958).

23) J. A. M. Smit, J. A. P. P. Van Dijk, M. G. Mennen, and M. Daoud, Macromolecules 25, 3585 (1992).

24) C. A. Mitchell, S. Kalies, T. Cizmár, A. Heisterkamp, L. Torrance, A. G. Roberts, F. J. Gunn-Moore, and K. Dholakia, PLoS One 8, e79235 (2013).

25) D. Müter, H. O. Sørensen, H. Bock, and S. L. S. Stipp, J. Phys. Chem. C 119, 10329 (2015).

26) A. W. Robards, Annu. Rev. Plant. Physiol 26, 13 (1975).

27) V. Hernández-Hernández, M. Benitez, and A. Boudaoud, J. Exp. Bot. 71, 768 (2020).

28) C. Faulkner, Curr. Biol. 28, R1374 (2018).

29) R. Raliya, C. Franke, S. Chavalmane, R. Nair, N. Reed, and P. Biswas, Front. Plant Sci. 7, 1288 (2016).

30) G. Zhai, K. S. Walters, D. W. Peate, P. J. J. Alvarez, and J. L. Schnoor, Environ. Sci. Technol. Lett. 1, 146 (2014).

31) E. Corredor et al., BMC Plant Biol. 9, 45 (2009). 